\title{
Bleeding in Plastic-Aesthetic Surgery: A Prognostic Pathway with Clinical Application
}

\author{
Stefano Avvedimento ${ }^{1} \cdot$ Gabriele Madonna $^{2} \cdot$ Adriano Santorelli $^{3}$
}

Level of Evidence $V$ This journal requires that authors assign a level of evidence to each article. For a full description of these Evidence-Based Medicine ratings, please refer to the Table of Contents or the online Instructions to Authors www.springer.com/00266.

Dear authors,

We are grateful for your interest and valuable comments on our recent publication [1]. We fully agree that the type of surgery requires consideration when assessing the bleeding risk. The risk of bleeding is influenced by the type of surgical intervention other than individual factors (patient comorbidities and medications). Increased complexity of a surgical procedure is associated with prolonged operative duration, perioperative bleeding, and long hospital stays [2]. Surgeries that are at higher risk of blood loss (e.g. extended abdominoplasty, large-volume liposuction) may require additional measures to modify/minimize existing risk factors for bleeding. On the other hand, patients undergoing low-risk procedures (cutaneous and those requiring shorter operative time) may benefit less from a preoperative assessment of bleeding risk through a validated questionnaire. Incorporating into the scale an "estimate procedural-specific bleeding risk" could add more

\section{Gabriele Madonna}

gabriele.madonna@yahoo.it

1 Plastic Surgery Unit, Casa di Cura Villa dei Fiori, Napoli, Italy

2 Istituto Nazionale Tumori IRCCS Fondazione "G. Pascale" Di Napoli, Napoli, Italy

3 Plastic Surgery, Academy of Aesthetic Sciences, Napoli, Italy information to the overall risk of bleeding. We recognize that more data are needed for the validation of a structured bleeding index and specific criteria must be fulfilled for acceptability and reproducibility. Randomized controlled trials are needed to validate any risk assessment scale, identify the potential consequences of an intervention, and provide the evidence necessary to support permanent implementation of these changes.

Unfortunately, there are critical issues (e.g. high cost, time demand) that make these types of studies hard to design and conduct [3].

\section{Compliance with Ethical Standards}

Conflict of interest The authors declare that they have no conflicts of interest to disclose.

Human and Animal Rights This article does not contain any studies with human participants or animals performed by any of the authors.

\section{References}

1. Marlino S, Cagli B, Vitale A, Boccia A, Avvedimento S, Madonna G, Santorelli A (2020) Bleeding in plastic-aesthetic surgery: a prognostic pathway with clinical application. Aesthetic Plast Surg 1:201-206

2. Thomas AB, Shammas RL, Orr J, Truong T, Kuchibhatla M, Sergesketter AR, Hollenbeck ST (1117e) An assessment of bleeding complications necessitating blood transfusion across inpatient plastic surgery procedures: a nationwide analysis using the national surgical quality improvement program database. Plast Reconstr Surg 143(5):1109e-1117e

3. Thoma A, Sprague S, Temple C, Archibald S (2008) The role of the randomized controlled trial in plastic surgery. Clin Plast Surg 35(2):275-284

Publisher's Note Springer Nature remains neutral with regard to jurisdictional claims in published maps and institutional affiliations. 